BULLETIN Bulletin hispanique

HISPANIQUE Université Michel de Montaigne Bordeaux

114-2 | 2012

Varia

\title{
Le théâtre de la douleur d'Angélica Liddell
}

\section{Béatrice Bottin}

\section{OpenEdition}

Journals

Édition électronique

URL : https://journals.openedition.org/bulletinhispanique/2163

DOI : 10.4000/bulletinhispanique.2163

ISSN : 1775-3821

\section{Éditeur}

Presses universitaires de Bordeaux

\section{Édition imprimée}

Date de publication : 31 décembre 2012

Pagination : 775-798

ISBN : 978-2-86781-855-4

ISSN : 0007-4640

Référence électronique

Béatrice Bottin, « Le théâtre de la douleur d'Angélica Liddell », Bulletin hispanique [En ligne], 114-2 |

2012, mis en ligne le 05 janvier 2016, consulté le 28 juillet 2022. URL : http://journals.openedition.org/ bulletinhispanique/2163 ; DOI : https://doi.org/10.4000/bulletinhispanique.2163 


\title{
Le théâtre de la douleur d'Angélica Liddell
}

\author{
BÉATRICE BotTIN \\ Université de Pau et des Pays de l'Adour
}

Angélica Liddell simpose par son univers baroque, mêlé d'autobiographie et de performance. Elle sáaplique à réveiller la sensibilité du spectateur, à le confronter à la réalité et, à son tour, elle invente le théatre de la douleur en incarnant la souffrance et en sublimant l'horreur.

Mots-clés: Thêâtre contemporain, mise en scène, dramaturgie, Angélica Liddell.

Angélica Liddell se caracteriza por su universo barroco, impregnado de autobiografía y de performance. Se esmera en despertar la sensibilidad del espectador confrontándole a la realidad y a su vez inventa el teatro del dolor al encarnar el sufrimiento y sublimando el horror.

Palabras claves: Teatro contemporáneo, puesta en escena, dramaturgia, Angélica Liddell.

Agelica Liddell stands out, thanks to her Baroque universe, rich with autobiographical elements and performances. She strives to rouse the spectators' emotions, to confront them with reality and in turn, she invents the theatre of pain, by incarnating suffering and transfiguring horror into something beautiful.

Key words: contemporary drama, staging, dramatic art, Angélica Liddell.

$\mathrm{L}^{\prime}$ édition 2010 du célèbre Festival d'Avignon a dévoilé à un public aussi bien averti que béotien, deux pièces de la dramaturge, metteur en scène et interprète espagnole, Angélica Liddell ${ }^{1}:$ La casa de la fuerza et El año de

1. Son véritable nom est Angélica González, patronyme qu'elle conserve à ses débuts, lorsqu'elle joue la comédie. Pour son travail de dramaturge et de metteur en scène, elle adopte le pseudonyme Angélica Liddell, lequel évoque Alicia Liddell, la petite fille dont s'inspira Lewis Caroll dans Alice au pays des merveilles. 
Ricardo ${ }^{2}$. Bien que deux de ses œuvres aient été traduites en français ${ }^{3}$ et malgré sa participation, en 2008, au festival Mira de Bordeaux avec La desobediencia : yo no soy bonita, elle demeurait jusqu’à présent méconnue, voire inconnue en France. Cette invitation au prestigieux festival créé par Jean Vilar représente, sans nul doute, une consécration pour cette artiste écorchée vive, talentueuse et jouissant en Espagne d'une reconnaissance dont témoignent les prestigieux Prix ${ }^{4}$ qui lui ont été attribués pour son travail dramaturgique et scénographique, une reconnaissance, à nos yeux, très largement méritée. Les deux spectacles présentés à Avignon appartiennent à deux processus de création bien distincts et n'ont absolument rien à voir l'un avec l'autre, si ce n'est que tous deux sont des "actes de résistance". Dans El año de Ricardo, Angélica Liddell réalise un impressionnant travail d'interprétation; elle y joue le rôle de Richard III, un monstre de cruauté. En revanche, dans La casa de la fuerza, il n'y a pas de jeu d'acteurs, pas de comédiens, mais des personnes bien réelles qui travaillent avec leurs propres ressentis, cherchant à survivre à la douleur et à la souffrance. Les spectateurs se voient confrontés aux horreurs qui caractérisent la société et sont invités à partager les sentiments exprimés sur scène, à les comprendre. Les deux montages ont reçu un accueil plus que chaleureux de la part du public même si, inévitablement, certaines âmes sensibles ont quitté la salle avant la fin des spectacles. La critique ne tarit pas d'éloges, encense la créativité et le talent de l'artiste et considère son théâtre comme un " électrochoc ». Le journal Libération $^{5}$ lui consacre une double page tandis que Télérama, Le Figaro, Le Monde, l'Humanite $e^{6}$ la présentent comme un grand nom du théâtre espagnol contemporain. Il n'en demeure pas moins qu'Angélica Liddell évolue dans le monde du théâtre alternatif dont la diffusion reste cantonnée aux Festivals et autres manifestations artistiques, comme le souligne César Oliva : " (el teatro alternativo) se distribuyó por festivales y muestras hasta conformar un auténtico circuito específico para públicos minoritarios, lejos de la común ambición de

2. La casa de la fuerza (2009), Cloître des Carmes, durée 5h entractes compris, du 10 au 13 juillet 2010, première en France. El año de Ricardo (2005), Chapelle des Pénitents Blancs, durée 2h, les 17,18 et 19 juillet, première en France.

3. Angélica Liddell, Et les poissons partirent combattre les hommes, (Traduction de Christilla Vasserot), Paris, Éditions théâtrales, 2008. Liddell Angélica, Belgrade, (Traduction de Christilla Vasserot), Paris, Éditions théâtrales, 2010.

4. En 2003, elle reçoit le Prix «Dramaturgia Innovadora de la Casa de América»; en 2004, le Prix SGAE pour Mi relación con la comida; en 2007, le Prix «Valle-Inclán de Teatro» pour El año de Ricardo, ainsi que le Prix «Notodo» du public pour son spectacle Perro muerto en tintorería: los fuertes.

5. R.S., "Comment transformer la douleur en autre chose? ", Libération, 06/07/2010.

6. Daniel Conrod, "Le beau bordel d'Angélica Liddell ", Télérama, 16/07/2010. Héliot Armelle, "Angélica Liddell, naturellement skakespearienne ", Le Figaro, 18/07/2010. Salino Brigitte, "Une Espagnole en colère. Angélica Liddell entre au Festival d'Avignon ", Le Monde, 13/07/2010. Sirach Marie José , "Angélica Liddell, Un ange noir parachuté au cloître des Carmes ", L'Humanité, 12/07/2010. "Angélica Liddell : "Le plateau est un champ de combat" ", entretien réalisé et traduit par Marie José Sirach, L'Humanité, 19/07/2010. 
cualquier empresa teatral de permanecer en cartel siquiera sea por unos pocos dias " . À vrai dire, ce mouvement underground se structure à partir de 1990 au cœur même de la Sala Pradillo de Madrid, un espace de travail et de recherches fondé et dirigé par Carlos Marquerie et Juan Muñoz. Des liens étroits se tissent entre les créateurs, donnant lieu à des collaborations inattendues. Ceux-ci croisent leurs expériences, leurs spécialités, pour monter des spectacles hybrides, preuves de l'émergence d'un nouveau courant théâtral. Pour certains artistes, cette enceinte consacrée aux nouvelles tendances de la scène espagnole marque le début d'un travail collectif qui, de nos jours, continue son chemin. Rodrigo García, Carlos Marquerie et Elena Córdoba en sont les exemples probants, dans la mesure où le premier confie les lumières de tous ses spectacles au second et fait régulièrement appel à la danseuse pour orchestrer les chorégraphies de ses mises en scène. Même s'il est incontestable qu'Angélica Liddell présente des similitudes avec ces créateurs, elle n'a pas participé à l'aventure de la Pradillo. Elle opte résolument pour la dimension luminotechnique que lui apporte Carlos Marquerie à l'occasion du montage de $Y$ los peces salieron a combatir, soit à partir de 2003 ; c'est la touche poétique supplémentaire dont elle ressent désormais la nécessité pour sa propre scénographie. Le parti pris esthétique de ces artistes ne correspond en rien aux programmations des théâtres publics qui affichent de nombreux spectacles de divertissement et témoignent du retour en force de la comédie musicale. Le public serait-il plus sensible aux comédies légères ? Aurait-il un regain d'intérêt pour les valeurs sûres du répertoire classique? De toute évidence, les spectacles qui font salle comble et qui restent à l'affiche plusieurs semaines sont quelque peu éloignés de cette génération de dramaturges, metteurs en scène et interprètes espagnols qui bousculent le discours politiquement correct et redéfinissent les codes de la traditionnelle représentation théâtrale. Les langages scéniques, tels que la danse, les arts plastiques s'entremêlent, se complètent, s'harmonisent pour mieux exprimer la réalité, la vérité, sur cet espace de liberté que devient la scène. Les installations vidéo se substituent aux décors, enrichissent l'action dramatique, complètent le texte, lequel n'est plus au cour de la représentation. Parfois, on ne le dit même plus, on le projette sur des écrans géants où il devient l'un des éléments du décor. En outre, les actions scéniques acquièrent une certaine indépendance et se détachent des dialogues et des monologues. Les auteurs ne se cantonnent plus au seul travail dramaturgique; ils se chargent de la mise en scène, conçoivent eux-mêmes les scénographies. D'étroites collaborations s'établissent entre ces derniers et les comédiens qui contribuent à la construction du spectacle et lui apportent parfois leurs propres mots.

Afin d'appréhender toute la portée de l'une des dernières créations d'Angélica Liddell, un éprouvant voyage de plus de cinq heures dans La casa de la fuerza, il est nécessaire de revenir sur le parcours de l'artiste, d'analyser ses processus

7. César Oliva, "Experiencias de un espectador experimental. Algunas ideas sobre la modernidad escénica en España en los albores del siglo XXI ", AA.VV, Tendencias Escénicas al inicio del siglo XXI, Madrid, Visor Libros, 2006. 
de création et de mettre en lumière les œuvres, tant littéraires qu'artistiques, qui influencent son travail dramaturgique et scénographique dont le point de départ est la douleur comme paradigme de la beauté.

Angélica Liddell est originaire de Figueras où elle naît en 1966. Fille unique d'un père militaire et d'une mère au foyer, elle fréquente jusqu'à l'âge de 17 ans, des établissements scolaires religieux qui lui laisseront d'amers souvenirs. Perturbée par les nombreux déménagements dus à la profession de son père, en proie à l'ennui et à la solitude, aussi bien au collège que dans l'enceinte des austères casernes où est logée sa famille, affaiblie par de sévères problèmes de santé, elle se réfugie dans l'écriture et commence à imaginer, dès l'âge de douze ans, des contes mettant en scène des monstres :

Soy una suicida sin suicidio. Un momento particularmente propicio para acabar con todo es la adolescencia. En esa época mi vida era un desastre debido a los continuos traslados a distintas ciudades que teníamos que hacer a causa del trabajo de mi padre, que era militar. Cuando tenía trece años nos fuimos de Valencia, donde viviamos en el campo y tenía a mis amigas, a Burgos. Fue una época complicada. Pasé la adolescencia entre médicos. Tuve una úlcera sangrante, algo muy inusual a esa edad, y sufri una fuerte depresión. Lo recuerdo como mi etapa más angustiosa y triste ${ }^{8}$.

Quelques années plus tard, elle reprendra dans ses pièces, ce genre littéraire ainsi que ces personnages et trouvera dans le théâtre un exutoire certain à son mal être. Elle affirme à ce sujet : "El teatro es lo que me impide pegarme un tiro ${ }^{9}$ ". Alors que son père la destine à une carrière militaire, elle s'y refuse et décide de s'inscrire à la Real Escuela Superior de Arte Dramático de Madrid (RESAD). Après une Licence d'Art Dramatique, elle poursuit des études de Psychologie à l'Université Autonome de Madrid.

En 1988, elle écrit sa première pièce, Greta quiere suicidarse. Pour la mise en scène, elle utilise des marionnettes "parce que je n'imaginais pas être un jour capable de travailler avec des gen ${ }^{10} »$. Elle ne renie pas ce spectacle comme elle l'a fait, il y a peu, pour son Frankestein ${ }^{11}$. Pour la première fois, elle aborde le thème du suicide, qu'elle reprendra dans des créations ultérieures : "Era malísima, pero todavia hoy sigo hablando del suicidio, quizá porque no me atrevo a quitarme la vida ${ }^{12}$ ». Grâce à ce texte, elle obtient sa première récompense, le Prix de la ville d'Alcorcón. Cependant, c'est en 1993 que démarre véritablement la carrière artistique d'Angélica Liddell, lorsqu'elle fonde à Madrid la compagnie Atra Bilis

8. Montero Javier. A partir de ahora. In Letras Libres. Site de la revue. [En ligne]. Letras Libres, 2011 [consulté le 2 octobre 2010]. Disponible sur: http://letraslibres.com/index. php?art $=12807$

9. Juan José Mateo, «El teatro es lo que me impide pegarme un tiro », El País, 27/12/2007.

10. Programme du Festival d'Avignon, propos recueillis par Christilla Vasserot, juillet 2010.

11. Pièce écrite en 1997 et mise en scène le 8 janvier 1998, à l'occasion du XIII Festival de Teatro Visual y Titeres de Barcelone. Ce spectacle, pour lequel Angélica Liddell et Gumersindo Puche manipulaient des marionnettes géantes, dénonçait un ordre social chaotique générateur de monstres.

12. Javier Vallejo, «Por las revueltas de Angélica Liddell », El País, 17/10/2009. 
Teatro pour laquelle elle écrit, met en scène et interprète ses propres textes. Au fil des années, la compagnie se resserre et ne repose désormais plus que sur deux artistes: Angélica Liddell et Gumersindo Puche ${ }^{13}$. Dans ces premières œuvres, lorsque la dramaturge joue la comédie, elle retrouve son patronyme d'origine, Angélica González. En revanche, pour identifier son travail dramaturgique et scénographique, elle recourt à son pseudonyme, emprunté à la jeune Alicia Liddell qui fut l'inspiratrice du récit Les Aventures d'Alice aux pays des merveilles de Lewis Caroll.

Ses spectacles s'articulent autour des thèmes de la famille, de l'enfance, de la guerre, de l'immigration, de la violence, de la folie, du suicide, de la souffrance aussi bien individuelle que collective. Des personnages de fous, de monstres, de marginaux, sont au centre de ses créations. Toutefois, progressivement la dramaturge propose un travail toujours plus autobiographique dont le moteur est sa propre indignation face au monde. Ses mises en scène gomment délibérément les frontières entre les arts. Pour ce faire, elle exploite les différents langages scéniques tout en imaginant de nouvelles formes d'expression. La parole et les mots sont relégués au second plan afin d'adopter un nouveau langage, celui du corps. Angélica Liddell conçoit la scène comme un espace de résistance, elle fait preuve d'un engagement absolu non seulement physique mais également moral. Elle libère ses passions, repousse les limites de la douleur et de la pudeur. Le public est pris à témoin, bousculé, dérangé, à tel point que certains spectateurs s'éclipsent. Ses premières pièces sont construites de dialogues mais peu à peu, elle privilégie les monologues et c'est seule en scène qu'elle affronte le public.

La jeune compagnie Atra Bilis fait ses débuts avec Eljardín de las Mandrágoras. Pequeña tragedia sexo-metafísica dividida en nueve escenas y cinco lirios (1993). Angélica Liddell y met en scène le suicide d'une femme par pendaison ; un évènement qui entraîne la descente aux enfers de son amant : "su amante decide, en una especie de metamorfosis, salir de la muerte de ella como si fuera de una crisálida. Él nace de la crisálida de la muerte de la mujer convertido en la mujer muerta y entonces utiliza a un muchacho para purgar todo su dolor». Sexe, mort, passion et fantaisie rythment l'action. Pour Angélica Liddell, "se trata de una creación dirigida a los sentidos tomando como referentes más directos la pintura y la música. Es una búsqueda dolorosa de la belleza, es decir, de lo insoportable ${ }^{14}$ ". Cette déclaration reflète parfaitement l'aspiration de la créatrice. La quête douloureuse de la beauté est sa seule fin et c'est en 1994, dans Dolorosa, qu'elle prend véritablement tout son sens. Les personnages s'y révèlent plus ancrés dans la réalité, éloignés d'un monde fantaisiste. L'œuvre retrace l'histoire d'amour tumultueuse entre une prostituée et son amant malingre et misérable, deux

13. Auteur, metteur en scène, interprète et Docteur en Littérature espagnole de l'Université Carlos III de Madrid.

14. Liddell Angélica. El jardín de las mandrágoras. In Parnaseo. Site de Documentation. [En ligne]. Universidad de Valencia, 1996 [consulté le 2 octobre 2010]. Disponible sur:

http://parnaseo.uv.es/ars/autores/liddell/mandragoras/jardin.html 
êtres en perdition, deux âmes en peine, en décalage. La jeune femme tombe systématiquement amoureuse de tous ses clients et se dit prête à mourir pour chacun d'eux. Quant à cet amant, il croule sous le poids du passé, du présent et du futur et n'aspire qu'à une chose, être aimé. Plus tard, dans Frankestein (1998), texte qui s'inspire de celui de Mary Shelley, les comédiens partagent la scène avec des marionnettes géantes qu'ils manipulent eux-mêmes. Angélica Liddell avoue ne plus s'identifier à cette œuvre qu'elle qualifie de "cursilada". Cependant, les mannequins utilisés ici pour la première fois occuperont une place de choix dans plusieurs de ses mises en scène futures où des poupées démembrées, des ours en peluche, des mannequins de cadavres et de pendues, envahissent l'espace scénique. En outre, dans cette pièce, apparaissent deux groupes de personnages : les manipulateurs et les manipulés, lesquels forment le traditionnel et inséparable couple, victime - bourreau que l'on retrouve dans toutes ses créations. L'ennemi est à la fois l'autre, la société et soi-même. En 1965, The Living Theatre montait déjà une version de Frankestein, à partir du roman de Mary Shelley. Julian Beck, l'un des fondateurs de la compagnie, insistait sur l'innovation de la pièce qui s'attachait à rendre l'horreur publique :

Ya sé que debe ser insólito que un teatro proponga hacer en Venecia una obra sin un texto establecido, pero tengo la esperanza de que a ustedes les emocionará, como nos emociona a nosotros, la perspectiva de una obra en la tradición de la idea de Artaud de un teatro no literario que, mediante el ritual, el horror y el espectáculo pudiera llegar a ser un acontecimiento teatral aún más válido que el Teatro verbal de Ideas, que por tanto tiempo ha dominado nuestros escenarios $^{15}$.

En outre, il revendiquait un théâtre hybride où le texte devenait modulable, à la limite du superflu. A l'instar de l'artiste américain, Angélica Liddell conçoit des spectacles qui n'ont pas pour fonction de sublimer le texte: "La escena no es el lugar de la realización de la palabra, sino el lugar de su acabamiento, de su final, de su muerte. En la escena, el texto va a morir ${ }^{16}$ ". En revanche, ils mettent en relief les capacités d'expression du corps et des arts plastiques et audiovisuels.

En 2000, dans La falsa suicida, la comédienne interprète une femme qui décide de mettre fin à ses jours en se jetant dans le vide. Elle est miraculeusement sauvée par un homme mais ce dernier se blesse et restera paralysé à vie. Ces deux événements, étroitement liés, vont changer le cours de leur vie. La jeune femme s'exhibe désormais dans un peep-show tandis que lui tente de survivre, d'accepter son handicap et d'évacuer sa colère en noyant des chats dans des piscines. Le destin voudra qu'ils se retrouvent quelque temps plus tard, dans le sex-shop où elle travaille : "Ofelia : Las mujeres desnudas somos como los muertos, nadie puede dejar de mirarnos. Horacio: Y yo matando gatos por tu culpa. El hombre del saco, el que ahoga animalitos en la piscina de tu rascacielo ". Ces

15. John Tytell, The Living Theatre Arte, exilio y escándalo, Barcelona, Los Libros de la Liebre de Marzo, 1999, p. 140.

16. Angélica Liddell, in Politicas de la palabra, edición de Cornago Óscar, Madrid, Espiral/ Fundamentos, 2005. 
individus que tout semble opposer se découvrent un nouveau point commun: tous deux fuient leur identité. Pour mieux y parvenir, ils choisissent d'en adopter de nouvelles, celles d'Horace et d'Ophélie, les personnages de Hamlet de Shakespeare qu'ils se mettent à jouer : "Si a ti te llaman Ofelia, me llamaré Horacio ». Dans une interview, Gumersindo Puche revient sur la complexité des personnages, en quête d'identité :

Horacio la reconoce en la sala de exhibición y comienza a ir a verla cada vez más a menudo, y echa moneda tras moneda para no perderla de vista mientras dura su show. En realidad es una historia de amor, aunque al principio lo unico que sienten al unir de nuevo sus destinos es un odio casi visceral. La falsa suicida no es una versión de la obra Hamlet, de Shakespeare. Toma la ambigüedad del personaje de Ofelia del cual no se sabe si se suicida o muere por accidente, y la extrapola a la historia de una mujer que no sabe verdaderamente quién es ${ }^{17}$.

Ils évoluent dans un sombre décor, une atmosphère sordide et macabre. Le plateau est envahi de poupées torturées, pendues par Horacio. Les comédiens utilisent des masques pour jongler avec les identités, se dévêtissent, se livrent à des corps à corps. Ils explorent les registres de la douleur, en offrent une interprétation esthétique tout en faisant tomber les barrières de la pudeur et des tabous. La comédienne joue, une bonne partie de la pièce, les pieds plongés jusqu'aux mollets dans un bocal en verre transparent rempli d'eau ; soit un emprisonnement qui, a fortiori, limite ses déplacements et symbolise l'enfermement spirituel de cette femme névrosée. Le but des comédiens est d'établir une distance entre le texte et les actions qui ne sont pas forcément liés. En revanche, ils placent le public dans une position de voyeur, semblable à celle occupée par Horacio, lequel passe son temps à se 'rincer l'œil' dans les peepshows. Pour ce faire, pendant plus de la moitié de la représentation, Angélica Liddell évolue complètement nue. Ces personnages marginaux, en proie à la folie, précèdent les monstres qui vont peupler le Triptico de la aflicción.

Dans les trois pièces qui le composent, El matrimonio Palavrakis (2001), Once upon a time in west Asphixia (2002) et Hysterica Passio (2003), Angélica Liddell s'attaque à l'un des fondements de la société : la famille. Elle façonne et interprète des monstres, des fous qui le sont devenus car dès leur naissance ils ont été maltraités. La première création raconte la descente aux enfers de parents dont la petite fille de sept ans a été sauvagement assassinée. Le plateau jonché de poupées estropiées et ensanglantées matérialise le crime et confère une ambiance lugubre à l'espace scénique. À la suite de cette tragédie ses parents sombrent définitivement dans la folie. Mais en est-elle véritablement la cause? Bien vite, le public comprend que ces deux êtres sont des névrosés, victimes de leurs propres géniteurs, des ogres, qu'ils ont essayé de combattre mais auxquels ils ressemblent. Le père se révèle responsable de la mort de la fillette avec la complicité de la mère qui s'évertue à clamer son innocence et préfère rejeter la

17. Pérez Gil Lila. El grupo Atra Bilis representa la negación de la propia identidad. In Parnaseo. Site de Documentation. [En ligne]. Universidad de Valencia, 1996 [consulté le 2 octobre 2010]. Disponible sur: http://parnaseo.uv.es/ars/autores/liddell/lafalsa/prensa/resenyas. html, consulté le 10/10. 
faute sur les autres. Le binôme Angélica Liddell - Gumersindo Puche interprète ces deux êtres en détresse, torturés, cruels mais aussi victimes. Dans Once upon a time in West Asphixia, deux adolescentes, fascinées par le parricide commis par l'un de leurs camarades qui s'est donné la mort et poussées par la haine qu'elles vouent aux adultes, commencent à planifier des crimes atroces qui les mènent progressivement à l'autodestruction. Enfin, le protagoniste de la dernière pièce, meurtri par les blessures physiques et psychologiques dont il a été victime toute sa vie, fait subir un violent interrogatoire à ses parents pour essayer de trouver des raisons de rester en vie. Hysterica Passio se déroule pendant la fête de Noël, traditionnellement célébrée en famille et habituellement joyeuse. Or, ici, la fête prend la tournure d'un rituel de cérémonie macabre. La magie de ce moment de retrouvailles disparaît complètement. Une célébration va néanmoins avoir lieu mais c'est celle de la destruction. L'image paisible du Père Noël est écorchée, anéantie. Tout d'abord par Hipólito, le maître de cérémonie pervers et sadique qui adopte son déguisement puis, par la destruction de la figurine à son effigie qui fond dans une casserole tout au long du spectacle. Hipólito présente ses parents (Angélica Liddell et Gumersindo Puche) au public avant de les soumettre à un interrogatoire auquel ils répondent par le biais de leur voix en off. Ils portent des costumes semblables à des soutanes; celui de la mère est grotesquement harnaché du symbole de la Croix Rouge. Ils ressemblent à deux sinistres prêtres sataniques, aux visages poudrés de blanc, au maquillage noir et rouge dégoulinant. Ils ont des airs de cadavres rappelant les personnages morts-vivants des films d'horreur. Ces parents indignes sont en réalité victimes de leurs ascendants et leur faute a été de reproduire ce qu'ils avaient au préalable vécu.

Angélica Liddell conclut cette réflexion sur la douleur, ses origines et ses conséquences, au sein de la famille, avec Lesiones incompatibles con la vida (2003), l'épilogue de ce triptyque. Dans cette pièce, il n'y a ni personnages ni fiction. Pendant un peu plus d'une vingtaine de minutes, Angélica Liddell, les pieds dans deux blocs de plâtre, se déplace difficilement le long d'un mur sur lequel sont projetées, en alternance, des images d'elle-même, de paysages urbains, de marginaux, de supermarchés. Dans sa main, elle tient une photo de famille qu'elle confronte, ici, aux réalités quotidiennes tout en exposant les raisons pour lesquelles elle a pris la décision de ne jamais devenir mère. Le texte débute ainsi :

A los hijos que no voy a tener.

No quiero tener hijos.

No quiero ir más lejos.

Soy una epidemia de resentimiento.

No quiero tener hijos.

Es mi manera de protestar. Mi cuerpo es mi protesta.

Mi cuerpo renuncia a la fertilidad.

Mi cuerpo es mi protesta contra la sociedad, contra la injusticia, contra el linchamiento, contra la guerra. 
Mi cuerpo es la crítica y el compromiso con el dolor humano.

Quiero que mi cuerpo sea estéril como mi sufrimiento.

Mi cuerpo es mi protesta ${ }^{18}$.

Une confession qu'elle reprend en $2007^{19}$, dans son spectacle $\mathrm{La}$ Desobediencia : yo no soy bonita où elle s'avoue victime d'un abus sexuel jusquelà tenu secret. Le montage mêle vidéo, monologues, musique et actions. Le corps, en tant que moyen d'expression et de contestation, subit la douleur, la rend concrète :

A partir de una experiencia personal me ensucio en el origen cotidiano, rutinario y tabú de la violencia sexual contra la mujer, que a menudo desemboca en violación y muerte. Es un miedo de nacimiento, podríamos decir que es algo así como una marca, un privilegio inverso, como si las niñas naciéramos con una letra escarlata pendiendo del vientre, un estigma que nos introduce en la ruleta rusa de las alimañas bárbaras. Acabar con la tiranía de la vergüenza, empleando la belleza, también es desobedecer.

La scarification est élevée au rang de rituel, devenant un auto-sacrifice nécessaire à l'expression de la douleur tout en lui conférant un effet de réel.

Un an auparavant, la maison d'édition Artezblai publie la trilogie intitulée Actos de resistencia contra la muerte. Le livre regroupe trois pièces : $Y$ los peces salieron a combatir contra los hombres (2003), Y cómo sepudrió Blancanieves (2005) et El año de Ricardo (2005). Dans la première, la dramaturge rend hommage aux Africains qui, à bord d'embarcations de fortune, tentent d'atteindre les côtes espagnoles. Nombre d'entre eux trouvent la mort pendant la traversée : c'est pourquoi, à force de se nourrir des cadavres de ces hommes, les poissons finissent par être dotés d'yeux humains. Sur le plateau, où s'entassent des bouteilles d'eau en plastique, se dresse une gigantesque croix faite de machines à laver le linge. La comédienne, vêtue d'une longue robe aux couleurs du drapeau espagnol, interprète le rôle d'une prostituée s'adressant à un Señor Puta (Gumersindo Puche), personnification de l'Espagne conservatrice mais dont le visage peint en noir, symbolise également les immigrés ayant trouvé la mort. Le texte fustige l'indifférence de la Péninsule Ibérique tandis que la mise en scène s'attache à exhiber le corps sans vie des clandestins qui n'ont pas droit à la parole. Gumersindo Puche incarne un homme auquel on aurait définitivement ôté la parole et la faculté de penser. Devenu muet, il est déshumanisé. Son corps dénudé subit la violence du personnage prénommé Angélica. Les acteurs incarnent la souffrance de ces êtres oubliés. La pièce se rapproche de l'art performance au moment où Angélica Liddell rompt le fil de la narration

18. Angélica Liddell, Tríptico de la aflicción/Lesiones incompatibles con la vida, Bilbao, Artezblai, 2012, p. 85.

19. La même année, la collection "Pliegos de Teatro y Danza», dirigée par Antonio Fernández Lera, édite La desobediencia : hágase en mi vientre, un titre regroupant cinq textes : Lesiones incompatibles con la vida (2003), Broken Blossoms (2004), Yo no soy bonita (2005), Enero (20062008) et Ni siquiera el fuego goza de buena salud (2007-2008). Ces œuvres ont été montées, soit sous forme de triptyque, soit indépendamment les unes des autres. Leur inspiration est très nettement autobiographique. 
pour revenir sur le processus de création du spectacle en s'appuyant sur une installation vidéo. La compagnie accorde une place de choix à l'improvisation, comme nous le verrons plus précisément dans La casa de la fuerza.

Le second volet du triptyque, explore le personnage de Blanche Neige dans $Y$ cómo se pudrió Blancanieves : "Hemos utilizado el personaje de los Grimm, Blancanieves, para concentrar sobre ella el horror de la guerra, la muerte, el castigo, la violación, el hambre, el odio, hemos convertido a Blancanieves en una niñsoldado, y lo asombroso es que sigue siendo una niñ $a^{20}$ ». La violence envers les enfants, les récits de guerre, les épisodes de brutalité et de cruauté font référence à des faits réels et vont bâtir le texte de la troisième pièce, El año de Ricardo. Angélica Liddell revisite le Richard III de Shakespeare et offre un spectaculaire travail d'interprétation, reposant essentiellement sur la comédienne. Celle-ci ressuscite le monstrueux monarque, représentant de "la podredumbre moral», dans son corps de femme qu'elle mène à la limite de la résistance physique. Elle insiste sur le pouvoir du corps à franchir la barrière des mots. Le personnage est accompagné de son fidèle serviteur Catesby qui ne dit mot. En parallèle, la comédienne évoque l'invasion de l'Afghanistan et de l'Irak, met des mots sur l'horreur, incarne le mal : "En El año de Ricardo el cuerpo es el territorio escénico. Es el cuerpo que encarna la deformidad espiritual de Ricardo ${ }^{21}$ ". Par le truchement de ce personnage, elle fait l'état des lieux de la société et de ses maux. Le discours est cru, violent, cinglant, pour mieux dénoncer les crimes de guerre contre l'humanité, les enfants sacrifiés, les hommes torturés, la complicité de la religion, la privation de liberté, les déviances du pouvoir :

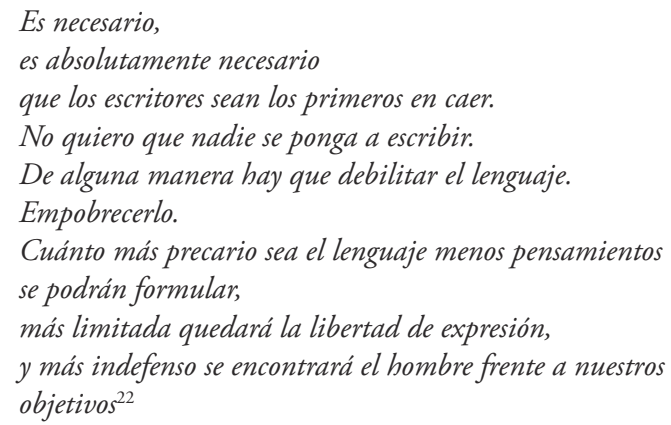

Sur le plateau, sont disposés des objets familiers : une soupière en porcelaine, un sanglier empaillé, de petites croix fixées sur une pomme de terre et un caillou, des fruits, des blocs de terre plantés de fleurs, etc. Au fond à gauche, on distingue la reconstitution d'une chambre à coucher. Suspendue au cintre, la photo retouchée d'un enfant nu, les bras en croix, martyr aux allures christiques des horreurs qui vont être énumérées sur scène. Non loin, se dresse un dessin

20. Óscar Cornago, "Angélica Liddell " in Politicas de la Palabra, Madrid, Espiral/ Fundamentos, 2005.

21. Conférence de presse du 15 juillet 2010, Avignon (non publiée).

22. Angélica Liddell, El año de Ricardo, Bilbao, Artezblai, 2009, p. 85. 
représentant deux enfants effrayés, en larmes et visiblement en fuite. Autant d'éléments faisant référence à l'Histoire et au présent.

En 2006, Gumersindo Puche et Angélica Liddell interprètent Boxeo para células y planetas. El miedo a la muerte como origen de la melancolia, honorant ainsi une invitation du Musée d'Art Contemporain de Castilla y León à participer à des journées ayant pour thème Lo siniestro y otros miedos contemporáneos:

Empleando la técnica del ensayo - ficción, presentamos la figura de Pascal Kahn, un personaje que experimenta un acontecimiento decisivo en su vida que destabiliza su existencia ordinaria hasta destruirla por completo. Pascal encuentra su nueva identidad a través de lo siniestro, a través del miedo a la muerte. Y este retrotraerse en el "yo" no sólo le permite reflexionar sobre su propio miedo sino sobre la naturaleza humana, de manera que el narcisismo procedente del temor a la muerte establece un vínculo terriblemente sólido con lo externo, es decir, con lo social, en definitiva con la tragedia de la humanidad. El miedo a la muerte, el deseo de ser eterno, sume al protagonista en una melancolía patológica de la que le resulta imposible escapar. La melancolía deja de ser un estado de ánimo transitorio para convertirse en una forma de vida, e incluso en una forma de pensamiento. El conflicto de Pascal Kahn tiene que ver con la contradicción que plantea "ser" al mismo tiempo "Hombre carne - hombre hueso" y "Hombre humanidad". De qué manera se puede compaginar la búsqueda individual de la felicidad con la catástrofe de lo humano. Para meditar acerca de este conflicto Pascal realiza una serie de "collages" donde conecta la ciencia con la guerra, las células con los planetas, los crímenes contra la humanidad con el descubrimiento de nuevas especies. De este modo hace un repaso estético del horror que define nuestros convulsos tiempos. La derrota interior de Pascal encuentra una conexión dolorosa con la derrota de la razón.

(NOTA: Los espectadores deberán traer consigo radiografias, medicamentos, analiticas, historiales clinicos... $)^{23}$

Elle imagine une mise en scène surprenante et inhabituelle puisque les spectateurs sont assis autour de tables formant un grand cercle. La configuration de l'espace scénique rend propice des face à face à quelques centimètres de distance, entre acteurs et spectateurs. Les rôles sont altérés, échangés, partagés. Le public n'est plus seul à regarder.

En 2007, dans Perro muerto en tintorería: los fuertes, elle met en scène deux couples dans une société du futur. Ces derniers n'ont plus d'ennemis. Quelque chose les effraie pourtant : ce qui est au cœur de leur tourmente c'est qu'ils en ignorent la cause. Le point de départ de la pièce n'est autre qu'une phrase issue du Contrat social de Jean-Jacques Rousseau : "La conservación del estado es incompatible con la conservación del enemigo, es preciso que uno de los dos perezca, y cuando se hace perecer al culpable es menos como ciudadano que como enemigo ".". La perfection du système dans lequel vivent désormais les personnages, dont le fondement repose sur la répression morale, réveille leurs instincts les plus primaires. La pièce débute par une violente insulte adressée par Angélica

23. Angélica Liddell, Programa de mano de Boxeo para células y planetas, La Porta Barcelona, 2006.

24. Angélica Liddell, Perro muerto en tintorería: los fuertes, Madrid, Nórdica Libros, 2008, p. 174. 
Liddell aux spectateurs " hijos de puta $»^{25}$. La dramaturge justifie cet acte par un désir de se rendre vulnérable, sans défense face au public, une mise en danger délibérée, réfléchie, mais aussi une illustration de la réplique de Jean-François Rameau, l'un des personnages de l'œuvre de Diderot, Le neveu de Rameau, lequel affirme: " autodegradarse es ser excelente en algo ${ }^{26}$ ":

El día 12 de agosto, un día antes de empezar los ensayos, estaba pasando por uno de los momentos más dolorosos de mi vida, apenas tenía fuerzas para desayunar, todavia hoy me sigue costando tragar comida. Tuve que empezar los ensayos asi, arrastrando unas ganas de morir insoportables. Día tras dia he trabajado con esas ganas de morir. A medida que arraigaba mi angustia la obra se iba volviendo más colérica, más demoniaca. Creo que he hecho pornografia del espiritu. Encontré una afinidad fundamental, identifiqué la derrota interior con la derrota de la humanidad. Sentía que el sufrimiento privado era tan fuerte como una alambrada, como un naufragio. He utilizado la angustia para alcanzar algo bello, algún tipo de justicia social relacionada con lo bello, algún tipo de redención. Seguramente he fracasado. En uno de los últimos ensayos, inconscientemente, me hice cortes en una pierna con una taza rota. Empecé a sangrar y nadie paró el ensayo hasta que el fluir de la sangre se hizo intolerable. La cicatriz que me ha quedado después de ese ensayo es Perro muerto en tintorería. El misterio de lo vivo y la encarnación de lo vivo 27.

Excédée par l'absence d'un "perro actor", qu'elle doit elle-même remplacer, Angélica Liddell, décharge sur scène sa colère en détruisant une chaise à coups de hache, avant de se glisser dans la peau de "el perro ", c'est-à-dire Jean-François Rameau. Sur le plateau les comédiens côtoient les mannequins de leurs propres cadavres et se livrent à un horrifiant jeu de destruction. Ils ne trouvent de remède à leur angoisse et à leur peur qu'en exacerbant leurs passions les plus sordides. Ils sont en quête de crimes, de violences sexuelles, de chaos. Pour survivre dans cette société, qui entretient de nombreuses similitudes avec celle de ce début $\mathrm{du} \mathrm{XXI}{ }^{e}$ siècle, et continuer à éprouver des sentiments, les personnages n'ont plus d'autre choix que celui de s'abandonner à l'auto-destruction en devenant leur propre ennemi. Angélica Liddell exprime sa colère contre le pouvoir, lance des diatribes contre les comédiens : "Nosotros los putos actores haciendo de putos perros ${ }^{28}$ " et se déclare en conflit avec le monde du théâtre qu'elle juge conservateur et réfractaire aux nouvelles formes de représentations :

La cúpula teatral, que podría pensarse que es progresista, excluye de manera premeditada cualquier nueva propuesta escénica. De hecho, se dedica a crear un estado de opinión en contra de toda forma de arte que signifique rebeldía. La cultura se ha vuelto simple entretenimiento vacio ${ }^{29}$.

25. Op. cit. (23), p. 3.

26. Op. cit. (23), p. 35.

27. Perales Liz. Angélica Liddell. In elcultural.es. Site du journal. [En ligne]. elcultural.es, 2006 [consulté le 2 octobre 2010]. Disponible sur: http://www.elcultural.es/version_papel/ ESCENARIOS/26009/Angelica_Liddell

28. S. Mayo María José. Angélica Liddell y su irritante experimento escénico. In elconfidencial.com. Site du journal. [En ligne]. elconfidencial.com, 2011 [consulté le 5 octobre 2010]. Disponible sur: http://www.elconfidencial.com/cache/2007/11/24/10_irritante_ experimento_escenico.html

29. Montero Javier. A partir de ahora. In Letras Libres. Site de la revue. [En ligne]. Letras 
Elle s'emploie à déconstruire les limites de la représentation, réveille le public, le violente et lance une ultime provocation lorsqu'elle pointe un révolver sur sa tempe et demande : " $i$ Algún hijo de puta se atreve a matarme?"

Dans ses dernières créations, Anfaegtelse (2008), Te haré invencible con mi derrota et La casa de la fuerza (2009), les personnages disparaissent pour laisser place à Angélica Liddell, laquelle s'exprime en son nom propre, s'employant « à rompre la barrière de la pudeur » et dévoilant son intimité la plus profonde. Le titre de la première qui signifie "danger, angoisse " en danois, en dit long sur le ton du texte et du spectacle. Kierkegaard s'était attaché à donner du sens à ce vocable dans Crainte et tremblement (Temor y Temblor), ouvrage duquel Angélica Liddell tire deux citations, destinées à ouvrir le spectacle et à expliciter son sens :

Cuando amamos entramos en guerra, es decir entramos en Anfaegtelse. Cada uno de nosotros será grande dependiendo de aquel con quien batalló.

Los guerreros antiguos luchaban en silencio, con la espada en una mano y secándose las lágrimas con la otra. Eso les hacía nobles. Lancelot camina, y sólo le vemos la espalda, es la posición más vulnerable, la más propicia para ser vencido ${ }^{30}$.

La comédienne évolue, ici, sur un plateau recouvert de charbon où trônent un métier à tisser et une table de mixage à laquelle sont branchés son baladeur MP4 et un micro. Sur le mur noir du fond, est projetée une phrase : "Bach en alemán quiere decir río". Angélica Liddell attribue un nouveau sens au patronyme du compositeur allemand et met sa musique à l'honneur. Elle la diffuse, l'écoute et interprète par deux fois Andante del concierto italiano. Une vidéo silencieuse, qui porte le titre du livre du philosophe allemand, est une autre déclinaison de l'angoisse : elle accompagne les actions scéniques. La dramaturge opte pour un récit autobiographique ; elle expose ses blessures, identifie ses démons. La souffrance se matérialise lorsqu'elle met son corps à l'épreuve en le salissant, en le grimant et en le violentant. Sans pudeur, elle lit des lettres d'amour de David, partage des séquences de son journal intime et pour finir, elle ose l'impensable en brodant la phrase, "Mamá te odio ", qu'elle signe de son sang.

La douleur et la souffrance atteignent leur paroxysme dans Te haré invencible con mi derrota qu'Angélica Liddell qualifie de "démence contrôlée", épuisante et déstabilisante. Elle rend hommage, ici, à la violoncelliste Jacqueline Du Pré, emportée par une maladie ravageuse à l'âge de quarante deux ans. Angélica Liddell se livre à une véritable prouesse d'actrice. Elle n'épargne ni son corps, ni son esprit, atteignant un douloureux état de transe. Le corps exprime la progressive descente aux enfers provoquée par la maladie et la souffrance qui l'accompagne. Sur scène, elle semble presque absente, dans un état second. Elle s'adresse directement à la musicienne : "Estoy aqui Jackie, no te dejo, no te

Libres, 2011 [consulté le 2 octobre 2010]. Disponible sur: http://www.letraslibres.com/index. php?art $=12807$

30. Angélica Liddell, Programa de mano de Anfaegtelse, La Noche en Blanco, Madrid, 13/09/2008. 
dejo, estoy aqui. " Elle cherche à établir une complicité et une communication presque surnaturelle avec cette dernière. Elle s'interroge à voix haute, le regard dans le vide, et apostrophe Dieu : "¿Por qué? Esa es la pregunta del dolor : ¿ por qué ? [...] Para ser feliz necesito la respuesta de Dios. Necesito pelear con Dios. Necesito los puños de Dios". Vêtue d'une longue robe blanche, elle ressemble à une figure christique, protagoniste d'une nouvelle passion. L'atmosphère intimiste n'en est pas moins pesante, la dégradation du corps et de l'esprit y est poussée à l'extrême. Ce montage est, sans aucun doute, l'un des plus éprouvant. Le spectateur n'en sort pas indemne car il assiste à une autodestruction dont la fonction est de révéler tout le pouvoir que le corps a sur l'esprit.

Ces dix sept années passées à écrire, mettre en scène et interpréter prennent tout leur sens dans La casa de la fuerza, pièce qui, sans nul doute, marque l'aboutissement du travail dramaturgique et scénographique de l'artiste. Dans cette œuvre, on retrouve ses thèmes de prédilection, essentiellement la douleur ainsi que la relation fétichiste qui s'est établie au fil des spectacles, avec les objets.

Le processus de création de La casa de la fuerza remonte au 2 octobre 2008, jour où l'artiste célèbre son anniversaire. En proie à un profond désespoir, Angélica Liddell trouve un soulagement partiel à son mal être en se réfugiant dans une salle de sport :

El día 2 de octubre de 2008, el día de mi cumpleaños, me sentía mal, estaba jodida por el paso del tiempo, y ya era plenamente consciente de que habia perdido todo lo que amaba o habia amado. Estaba asustada, furiosa y triste. Prácticamente habia dejado de leer y escribir. Ese mismo día, el 2 de octubre, me apunté a un gimnasio, el lugar de la fuerza y la resistencia, buscando algún tipo de contradicción o alivio. $Y$ alli empezó La casa de la fuerza.

Descubri que la extenuación fisica me ayudaba a soportar la derrota espiritual ${ }^{31}$.

Dans cette œuvre résolument autobiographique, la dramaturge poursuit son exploration de ce qu'elle appelle "la pornografía del alma". Elle opte, une fois encore, pour formuler l'innommable, exhiber l'inconfessable. Elle s'inspire également des Trois sours de Tchekhov à laquelle elle emprunte des répliques, clairsemées au fil de monologues émouvants. En outre, un workshop organisé au Mexique apporte l'ultime pierre à l'édifice et lui redonne envie de travailler avec des comédiens :

Por otra parte, el segundo viaje a México fue definitivo. Efectivamente, incluso el comentario más banal acaba culminando en acción. Del mismo modo que los chistes de judios culminan en Auschwitz, las rutinas de desprecio hacia la mujer culminan en feminicidio. La humillación cotidiana culmina en las muertes de Juárez, Chihuahua, y en unas leyes deterioradas por la misoginia ${ }^{32}$.

Angélica Liddell rend hommage aux femmes malheureuses, humiliées, violées, assassinées en croisant leur souffrance avec la sienne. L'artiste choisit

31. Angélica Liddell, Programa de mano de La casa de la fuerza, Festival de Otoño de Madrid, 2009.

32. Op. cit. (30). 
de s'entourer de deux comédiennes espagnoles, trois interprètes mexicaines, un groupe de Mariachis, un ami violoncelliste et l'homme le plus fort d'Espagne. Avec eux, elle s'enferme dans la maison de la force et y invite le public. Ce lieu où s'expriment solitude, humiliation, douleur, frustration, horreur et indignation, apparaît peuplé d'êtres malaimés qui refusent néanmoins de se résigner. Cette tragédie moderne, en trois parties, atteint la catharsis - évoquée par Aristote - en rappelant la mise en accusation de l'État du Mexique par la Cour Interaméricaine des Droits de l'Homme, laquelle reproche à ce dernier de ne pas veiller à la protection du droit des femmes à vivre libres et respectées. Enfin, la lecture d'une liste de noms de femmes, victimes de la violence machiste en Espagne suscite une prise de conscience collective et définitive.

Au début de la représentation, sur le plateau du Cloître des Carmes, on distingue un petit avion rose à pédales, des micros sur pied, des haltères, des caisses de bière Mahou, des fleurs, une table et trois chaises avec, à leurs pieds, des citrons. Au fond, sous les voûtes, on aperçoit des sacs de charbon. Côté cour, assises dans la pénombre, les comédiennes assistent en silence à l'arrivée des spectateurs. Puis, entrent en scène Getsemaní de San Marcos et Lola Giménez; elles s'attablent. L'une est vêtue d'une robe de couleur rose, l'autre de couleur bleue. Des tenues de bal, semblables à celles que l'on confectionne au Mexique pour les Quinceañeras. Une fillette s'avance; elle prend place au centre du plateau pour y lire une phrase écrite sur une feuille de papier: "No hay cerro, ni selva, ni desierto que nos libre del daño que los otros preparan para nosotros ". La petite fille regagne alors son petit avion, monte à bord, traverse la scène et disparaît. Les adultes se retrouvent désormais entre eux. Le public est invité à voyager au Mexique, à pénétrer dans la maison où il va être confronté à une véritable épreuve de force. Les corps des spectateurs vont inévitablement éprouver des courbatures à cause de l'exigüité des sièges sur lesquels ils seront assis pendant plus de cinq heures. En outre, ils subiront un véritable choc émotionnel.

D’emblée, par cette phrase lue par une enfant, Angélica Liddell pointe un doigt accusateur sur autrui, le responsable du mal et du désastre. Soudain, les deux comédiennes se lèvent, enlèvent leur culotte et s'allongent sur le sol. Leurs corps, sans aucun signe de vie, incarnent ceux des femmes que l'on a violées puis assassinées. Le public assiste à une première évocation de la violence : le silence pesant en dit long sur les circonstances du crime et la cruauté qui l'accompagne. Ici, les mots sont superflus, inutiles ; l'expression corporelle se substitue à eux. Les actrices se relèvent, retournent à la table où les rejoint Angélica Liddell, vêtue d'une robe noire semblable à un vêtement de deuil. Tout au long de la première partie, les trois femmes font part de leurs sentiments les plus personnels, évoquent de douloureuses et tumultueuses relations amoureuses. Ces confessions sont destinées à opérer un processus d'identification entre elles et le public. Ce sont des personnes bien réelles qui offrent leurs témoignages. Elles fument cigarette sur cigarette, boivent une bière puis une autre, se battent, rient, s'embrassent, tandis qu'elles se racontent 
les violences dont elles ont fait l'objet. Ici, on ne ment pas, on dit la vérité à voix haute et on fête des retrouvailles. L'ambiance semble festive mais la tension n'en est pas moins palpable. Ni l'entrée en scène de l'orchestre de mariachis, ni les plaisanteries échangées entre les hommes et les trois femmes, ne parviennent à détendre l'atmosphère plombée par la force des monologues et le choc des actions. Angélica Liddell interpelle les musiciens pour leur parler du rôle de Clint Eastwood dans L'Inspecteur Harry : "Pues Clint Eastwood en Harry el sucio-que me encanta esta peli-pues dice: disparar no es malo siempre y cuando mates al tipo que se lo merece ". Elle invite ses partenaires ainsi que le public à méditer sur cette réplique qui fait l'éloge de la violence et de la vengeance sanglante. Entre récits et longs silences, le public est immergé dans le drame de ces femmes blessées par l'amour, rêvant d'évasion. Les trois comédiennes tentent de recréer l'ambiance d'une cantine mexicaine et transforment la célèbre exclamation, "Vámonos a Moscú ", réitérée par Macha, Irina et Olga, dans le drame de Tchekhov, en "Vámonos a México ". Les boléros et les rancheras interprétés par les mariachis et repris par les trois comédiennes, ne parviennent pas à créer une ambiance joyeuse. Les trois femmes, à l'instar des trois sœurs, sont en proie à une immense détresse, plongées dans la solitude, le chaos ; leurs illusions sont en train de s'envoler. Le temps s'écoule sans qu'il se passe rien d'apparemment important. Elles essaient de se distraire, travaillent leurs muscles en soulevant des haltères, se soutiennent aussi bien physiquement que moralement. Les courts moments où la bonne humeur semble de retour, sont bien vite anéantis par les récits à la première personne par le biais desquels les actrices s'expriment. Dans cette pièce, il n'y a pas de personnages, pas de lieux précis, "hay vacios. Desolación $n^{33}$ ". Angélica Liddell le revendique et se dit fière du travail accompli avec les comédiennes : "Las actrices fueron capaces de romper con el pudor y han puesto su debilidad y su dolor sobre el escenario pues, gran parte de los fragmentos del guión fueron creados por ellas mismas basándose en tremendas historias personales". Les comédiennes dévoilent leur intimité au propre comme au figuré, se mettent à nu et mettent à nu leurs expériences personnelles. À ce sujet Getsemaní de San Marcos précise : "No se trataba de identificarse con la vida o con el dolor de Angélica. Se trataba de buscar en nosotras ese dolor [...], hablar de la violencia a través de una relación de amor, [...] rebuscar en una misma el abuso de poder ". Il s'agit de mettre à mal les limites du théâtre et de la représentation et d'assister à la déconstruction du personnage, lequel se confond avec le comédien. Cette première partie se caractérise par un rythme saccadé. Les actions, qui parfois s'éternisent, fonctionnent en parallèle avec le texte. Les moments d'attente sont suivis de scènes chorégraphiées, proches du théâtre-danse.

33. Perales Liz. Angélica Liddell "Tengo una inclinación natural a hablar de la parte podrida de las cosas”. In elcultural.es. Site du journal. [En ligne]. elcultural.es, 2006 [consulté le 20 octobre 2010]. Disponible sur: http://www.elcultural.es/version_papel/ESCENARIOS/26009/ Angelica_Liddell 
Le deuxième acte s'ouvre sur l'interprétation du Cum dederit de Vivaldi par Pau de Nut, accompagné de son violoncelle. La voix cristalline du chanteur et la magie de la musique enveloppent le spectacle d'une atmosphère paisible, presque solennelle. Toutefois une rupture s'opère, presque brutalement, lorsqu'Angélica Liddell prend la parole et projette une vidéo intitulée Venecia ${ }^{34}$ sur un écran disposé sur le plateau. La structure narrative des récits est celle d'un journal intime. La dramaturge commente les images de la chaîne CNN, au moment des bombardements israéliens sur la bande de Gaza ; en parallèle, elle évoque une relation amoureuse tumultueuse, suivie d'une violente rupture qui la pousse à se réfugier à Venise, qu'elle choisit de partager de nouveau, avec le public :

En enero me marché 10 días a Venecia a escribir. Coincidieron dos cosas: que yo estaba verdaderamente jodida y los ataques brutales de Israel a Palestina. Quise poner a prueba mi soledad ofreciendo sexo gratis en un chat (ofreciendo todo lo que puede ofrecer una puta) al mismo tiempo que las noticias de la CNN emitían la guerra en directo.

Angélica Liddell revient sur les violences qu' elle a subies de la part de l'homme qu'elle aimait. Puis, elle raconte, sans ambages, ses rapports sexuels virtuels avec des inconnus, sur fond d'images de guerre. Son discours s'affranchit de toute forme de pudeur ; ses sentiments sont exposés, voire exhibés. Semblables relations virtuelles ne lui procurent absolument aucun plaisir. Elles la plongent de nouveau dans la violence machiste et elles révèlent toute la bestialité de l'homme, ce mâle dont l'appétit sexuel inassouvi instrumentalise la femme, la souille, l'avilit. Elle crie, hurle, jure, blasphème, pleure, lance des invectives au public. La douleur, aussi bien celle de l'artiste que celle des victimes de la guerre, devient universelle, presque palpable, notamment lorsque l'artiste se scarifie choisissant comme fond sonore un titre du groupe pop espagnol La Oreja de Van Gogh (Dulce locura), dont toute la portée n'aurait assurément pas été totalement appréhendée sans ce spectacle. Car la musique est un élément fondamental dans les mises en scène et Angélica Liddell la sélectionne avec soin, toujours en lien avec le texte, la vidéo et les actions, en cohéence avec la narration. La comédienne reprend le refrain de la chanson faisant référence à une histoire d'amour violente et tourmentée suivie d'une douloureuse séparation. Puis, Angélica Liddell éponge le sang qui coule de ses genoux avec des mouchoirs blancs qu'elle dépose sur le visage des deux comédiennes étendues sur le sol : nouvelles illustrations des victimes, elles incarnent deux martyrs, deux figures christiques. Cette scène entretient des similitudes avec l'agonie du Christ au Jardin des Oliviers. Lola et Getsemaní, inertes, gisent sur le plateau tandis qu'Angélica semble se préparer à la mort. Un second

34. Ce spectacle ou plus exactement son ébauche avait fait l'objet d'une représentation en mars 2009, à l'occasion du Festival LP de Barcelone: "Era una manera de identificar la derrota personal con la derrota de la humanidad. De fondo se encontraba "la fuerza". De ese viaje surgió un diario y un texto. Y eso es lo que traigo. Y Pau tocará y cantará a Vivaldi, un veneciano ilustre que fue enterrado en una puta fosa común. Si, las fosas deberian ser comunes, ya que no nos reconciliamos en vida que se reconcilien nuestros jodidos gusanos. " 
titre du groupe, Muñeca de trapo, hommage aux femmes maltraitées par leur compagnon, envahit l'enceinte du Cloître. Il rythme la course circulaire effrénée d'Angélica Liddell à laquelle se joint Lola. Cette énergie, presque anormalement déployée, caractérise le monde du travail dans lequel les trois femmes se voient désormais plongées. Elles interprètent trois femmes croulant sous le poids des tâches imposées par la société, qui vont les engloutir : "Hay que trabajar. El trabajo se revela como una forma de aniquilación $n^{35}$ ". Avant de se lancer dans une incroyable débauche d'énergie, Angélica et Lola se soumettent chacune à une prise de sang réalisée par une infirmière sur le plateau. Armées de leur seringue remplie de leur propre sang, elles les vident sur leur chemise et sur celle de Getsemaní, du côté gauche. Elles déclarent ensuite : "Cada una de tus palabras es como un tiro en la cabeza pero me sangra el corazón". Blessées par l'amour, le cœur brisé, elles vont cependant déployer toute la force qui leur reste pour survivre encore un peu.

Les comédiennes accomplissent d'impressionnants efforts physiques. Sur la totalité du plateau, elles installent une dizaine de canapés sur lesquels elles disposent des bouquets de fleurs. Ces cercueils à ciel ouvert symbolisent l'avilissement, l'épuisement, que provoque le travail chez l'homme, jusqu'à son anéantissement. Puis, chacune d'entre elles revêt une robe d'un blanc virginal, avant d'aller chercher de lourds sacs de charbon qu'elles déversent sur scène. Un vêtement peu approprié aux circonstances, en décalage avec l'action, comme pour mieux souligner les aberrations du monde. Leurs robes d'une blancheur immaculée commencent à se couvrir de poussière noire. Une fois les sacs vidés, elles déplacent le charbon de quelques misérables mètres, à coups de pelle. Ereintées, Lola et Getsemaní s'écroulent sur le tas de charbon qui peu à peu les ensevelit. Gagnée à son tour par l'épuisement, Angélica s'arrête pour observer le désastre : "Ya no puedo más. Jamás iremos a México. No comprendo cómo vivo todavía, cómo no me he matado ». Elle reprend les mots d'Irina, lesquels accompagnent son dernier souffle. Par le côté cour, entrent discrètement Perla, María et Cynthia. Elles dégagent délicatement les trois corps et quittent discrètement la scène sur laquelle s'est accumulée de "la matière morte" : "la materia muerta como el carbón y elementos pesados, muy pesados sirven como vehiculo para que los espectatadores identifiquen mis sentimientos ${ }^{36}$ ". De nouveau, ici, les symboles sont gros de sens. Avec les éléments et les objets du décor, ils participent à la narration et se substituent aux mots.

La troisième partie s'ouvre sur un décor à la fois paisible et macabre. On pénètre dans une sorte de cimetière fleuri où trônent onze croix de couleur rose ; des robes sont suspendues à trois d'entre elles. Ces croix roses, "símbolos universales, iconos del dolor", commémorent les femmes disparues tout en symbolisant la rédemption à laquelle devrait aboutir ce voyage. Côté cour, on

35. Irina.

36. Lorasque Ángel. Angélica sin vergüenza. In larazon.es. Site du journal. [En ligne]. La Razón, 2012 [consulté le 4 octobre 2010]. Disponible sur: http://www.larazon.es/noticia/ angelica-liddell-sin-verg-enza 
distingue une voiture semblable à un corbillard accidenté, rempli de fleurs. Le spectateur est plongé dans la cruelle réalité du Mexique. À Ciudad Juárez, dans l'état de Chihuahua, il a fallu avoir recours à un néologisme, le "féminicide ", afin de qualifier la violence machiste qui s'est abattue sur les femmes depuis les années 90. La parole est donnée aux trois comédiennes mexicaines auxquelles on confie la tâche de guider la fin de ce voyage. Au préalable, elles lavent les pieds des actrices espagnoles. Telles de nouvelles Marie Madeleine, elles prodiguent religieusement des soins à leurs semblables espagnoles, les élevant au rang de la figure christique sacrifiée. Ce moment solennel de partage, de soutien et de respect précède leurs douloureuses confessions qui ne sont autres que des bribes de leur propre vie. Dans cette dernière partie, les victimes ont un nom, elles sortent de l'anonymat, elles confessent la peur, l'horreur, lèvent le voile sur l'innommable. Bien vite, on prend conscience de l'empathie qui s'est établie entre les trois comédiennes et Angélica Liddell. À plusieurs reprises, cette dernière s'est déclarée "resistente civil". À son tour, María déclare vouloir désobéir. Elle imagine le futur dans deux cents ou trois cents ans comme le faisait Verchinine dans la pièce de Tchekhov. Elle rêve d'un monde peuplé par une race d'hommes affaiblis, fruits de l'inceste devenu loi et dont Jocaste serait la reine. Une union définitive se scelle entre les comédiennes qui se retrouvent autour du violoncelliste pour célébrer un moment de communion. Telles des nymphes auprès de leur dieu, elles le couvrent de fleurs pendant qu'il chante Love me tender. Pau de Nut identifie son personnage au fantôme du monstre qui est pardonné : "Las actrices le transforman en cadáver exquisito cuando me cubren de flores; le invitan a la redención ${ }^{37}$ ". À la fin, l'homme le plus fort d'Espagne ${ }^{38}$ sort de l'ombre des coulisses, tel un monstre surgissant des ténèbres. Après avoir exécuté deux épreuves de force : renverser le véhicule et soulever une énorme pierre ronde, il s'allonge sur un canapé. Les six femmes l'entourent et disposent sur son corps des figurines en pâte à modeler rose, des reproductions plastiques de cadavres. Il est désigné coupable ; il porte sur son corps la souffrance des femmes, une manière de l'inviter lui aussi, à la rédemption.

De toute évidence, La casa de la fuerza se nourrit des années des créations précédentes. Or, cette fresque tragique n'en est pas moins dotée d'originalité et d'innovation. Elle s'inscrit dans la lignée de dramaturges et de metteurs en scène, influencés par Tadeusz Kantor pour qui : "El objetivo del teatro no es crear una ilusión de la realidad contenida en el drama. La realidad del drama debe realizarse en el escenario ${ }^{39}$ ". Sa conception de l'art théâtral et ses réalisations trouvent une résonnance dans les créations dramatiques d'Angélica Liddell où elle s'applique à manipuler la matière brute, les objets, à utiliser le corps ainsi que les multiples procédés techniques et artistiques afin de construire un langage destiné à exprimer toute son indignation et par ce biais, une identité

37. Rencontre avec le public, Avignon, 17/07/2010 (non publiée).

38. Juan Carlos Heredia, "El Porrúo", champion d’Espagne de "strongman".

39. Tadeusz Kantor, Teatro de la muerte y otros ensayos 1944-1986, Barcelona, Alba Editorial, 2010. 
théâtrale. Bien que le théâtre soit pour elle une forme d'exutoire, son objectif n'est pas de se substituer à "una consulta de psiquiatría ni ser una terapia de psicoanálisis. Quien necesite algo asi que se vaya mejor al médico ${ }^{40}$."

Dans Le théâtre et son double, Antonin Artaud conceptualisa le Théâtre de la Cruauté qu'il n'eut pas l'occasion de mettre en scène. À n'en pas douter, Angélica Liddell en nourrit ses créations et à son tour invente le théâtre de la douleur. Elle ne prétend donner de leçons à personne; ses diatribes ne proposent pas non plus de solutions. Elle s'impose par son univers à la fois baroque et mêlé d'autobiographie et de performance. Elle s'applique simplement à réveiller la sensibilité du spectateur, à le confronter à la réalité comme le préconisait l'auteur français :

C'est pour prendre la sensibilité du spectateur sur toutes ses faces, que nous préconisons un spectacle tournant, et qui au lieu de faire de la scène et de la salle deux mondes clos, sans communication possible, répande ses éclats visuels et sonores sur la masse entière des spectateurs $^{41}$.

Ce qu'elle revendique, à son tour, c'est une prise de conscience de la part du public, aussi s'efforce-t-elle d'incarner la souffrance, de sublimer l'horreur. Elle ne prétend pas apporter de réponse définitive à l'éternelle question sur l'utilité et la fonction de l'art. En revanche, elle s'emploie à démontrer que son parti pris de surexposer la douleur est un élément indispensable à sa quête de la beauté : "Por muy horrible lo que uno esté contando, el resultado estético siempre acaba siendo algo hermoso y es lo que nos permite darnos cuenta del horror y lo que nos permite darnos cuenta del mal es la lucidez del malvado $"{ }^{42}$. Artaud alléguait que « tout ce qui est dans l'amour, dans le crime, dans la guerre, ou dans la folie, il faut que le théâtre nous le rende, s'il veut retrouver sa nécessité " ${ }^{43}$. Ce sont précisément les thèmes qu'Angélica Liddell développe dans ses créations. C'est la manière poétique qu'elle convoque pour exprimer la violence du monde mais également pour exalter l'esthétique du laid, du sale, du sordide. Avec elle on assiste à un retour de la tragédie grecque et de la catharsis. Les sentiments que, par identification, le spectateur est amené à éprouver, tels que la pitié, la crainte, voire même la terreur, sont destinés à opérer une purgation. Les textes de la dramaturge se nourrissent de ses nombreuses lectures et se révèlent d'une astucieuse intertextualité. Elle utilise les mots des grands auteurs de la Littérature, les interprète, leur donne vie. Elle met un point d'honneur à rendre hommage aux écrivains illustres : parmi eux, entre autres, les philosophes grecs, ceux des

40. Lorasque Ángel. Angélica Liddell sin vergüenza. In larazon.es. Site du journal. [En ligne]. La Razón, 2012 [consulté le 18 octobre 2009]. Disponible sur: http://www.larazon.es/noticia/ angelica-liddell-sin-verg-enza

41. Artaud Antonin, Le théatre et son double, Paris, Gallimard, 1964, p. 148.

42. Liddell Angélica. Conférence du 15 juillet 2010, Avignon. In Théâtre Contemporain.net. Site de Ressources Théâtrales. [En ligne]. Cris (Centre de Ressourses Internationales de la Scène), 1998 [consulté le 24 septembre 2010]. Disponible sur: http://www.theatre-video.net/videos/ artiste/LIDDELL-Angelica?from=auteurs

43. Op. cit. (40), p. 132. 
Lumières, les dramaturges du théâtre élisabéthain, les auteurs anglo-saxons. Elle ne cesse de leur emprunter des citations qu'elle glisse parmi ses propres propos. En mai 1933, dans Le Théâtre et la cruauté, Artaud écrivait : "C'est pourquoi, autour des personnages fameux, de crimes atroces, de surhumains dévouements, nous essaierons de concentrer un spectacle qui, sans recourir aux images expirées des vieux Mythes, se révèle capable d'extraire les forces qui s'agitent en eux ". Angélica Liddell revisite les personnages des contes, ressuscite les ogres, réinvente le personnage de Blanche Neige et réinterprète les héros de la Littérature, tel Richard III. Elle met également en scène des chefs-d'œuvre de la Peinture et comme elle, elle a recours aux symboles, parfois difficilement déchiffrables au premier abord. Faisant siennes les théories d'Artaud, elle crée des œuvres qui entretiennent de nombreuses similitudes avec celles du Living Theatre notamment à travers l'idée que l'Art et la vie sont indissociables. Cette compagnie, qui fit scandale et qui révolutionna la mise en scène, se consacra à repousser les limites de la représentation en privilégiant le registre de la douleur. Les scénographies originales et inhabituelles plongeaient le spectateur dans un cérémonial au cours duquel il devait se rendre compte de l'horreur et de la douleur qu'il avait assimilées à son insu et à son corps défendant. En 1959, les personnages hérö̈nomanes de The Connection confiaient au public leur désarroi, leur solitude, avant de se piquer sur scène. Par le biais de ce montage, Judith Malina et Julian Beck désiraient provoquer une prise de conscience collective et une empathie avec les êtres faibles ou plus exactement affaiblis par la société :

Estos individuos, la hez de la sociedad, según se les consideraba, eran humanos, capaces de sentir y hablar de forma profunda y conmovedora, dignos de nuestro interés y respeto; teníamos que mostrar que todos teníamos necesidad de pincharnos y que lo que los drogadictos habian llegado a ser no era el resultado de una personalidad intrinsecamente mala, sino que era sintomático de los errores del mundo entero ${ }^{44}$.

À ces fins, dans La casa de la fuerza, Angélica Liddell donne la parole aux femmes humiliées tandis que les récits autobiographiques occupent progressivement une place prépondérante dans ses spectacles où son investissement physique et émotionnel est absolu. Un engagement similaire caractérisait celui des comédiens du Living Theatre qui utilisaient leur corps et différents langages artistiques, pour évoquer la violence et la souffrance. Seven meditations on Political Sadomasochism ${ }^{45}$ en est une illustration parfaite. La pièce reprend le monde délétère, méphitique de The Legacy of Cain ${ }^{46}$, qui s'appuyait sur l'ouvrage éponyme de Leopold Von Sacher-Masoch. Les personnages de victimes sont au centre de l'action dramatique. Les hommes sont maltraités,

44. John Tytell, The Living Theatre, Arte, exilio y escándalo, Barcelona, Los Libros de la Liebre de Marzo, p. 105.

45. L'œuvre évoquait les conditions de vie difficiles des prisonniers politiques. Elle a été représentée au printemps 1973 à l'Université de Caroline du Nord.

46. En 1970, Judith Malina et Julian Beck commencèrent à travailler sur un nouveau projet qu'ils intitulèrent The Legacy of Cain, inspiré du Legs de Caïn de Leopold Von Sacher-Masoch, publié à Paris, en 1874, chez Hachette. 
torturés, leur chair est mise à nu, ils agonisent pendant qu'on lit une liste de faits divers réels, publiés par Amnesty International. Angélica Liddell s'appuie également sur des faits divers réels et elle rencontre des membres d'associations venant en aide aux victimes. Elle relie ces aberrations aux crimes de l'Histoire, aux droits humains bafoués au nom de la religion.

La créatrice opte pour la séparation des mots et des actions, privilégie le collage des langages scéniques, et transforme ses montages en de véritables rituels. Elle se délecte en mettant en scène le « je », joue les premiers rôles de tous ses spectacles et parvient ainsi à construire un personnage qui lui est propre. Les humiliations et les aberrations de la vie quotidienne se matérialisent et prennent corps sur scène, d'une part dans les éléments du décor, d'autre part dans le jeu des comédiens. Les poupées démembrées qui jonchaient le plateau de la pièce El matrimonio Palavrakis faisaient référence, en premier lieu, à l'assassinat de la petite Cloé et par extension, aux infanticides en général. Le charbon symbolise le travail ainsi que l'aliénation à laquelle il conduit. Les marionnettes et les mannequins sont un prolongement du corps des acteurs, une incarnation de la putréfaction et de la mort. Les spectacles se caractérisent par l'omniprésence de la nudité, laquelle frôle l'exhibitionnisme mais devient nécessaire et porteuse de sens. La comédienne s'automutile, se détruit physiquement et spirituellement alors que, paradoxalement, elle considère le théâtre comme un moyen de survie. En guise d'introduction à la mise en scène de La desobediencia, elle écrit :

En una sociedad misógina, androcentrista y patriarcal, las mujeres se dividen en tres: virgenes, paridoras y putas. Mi cuerpo se convierte en una agresión contra la sociedad. Mi cuerpo se convierte en protesta. Es un acto de desobediencia. Castigo mi propio cuerpo para desobedecer. Me autolesiono para revolverme contra las lesiones que causa el rol que nos han impuesto desde el nacimiento. Utilizo la violencia poética para defenderme de la violencia real.

A partir de una experiencia personal (un abuso sexual aparentemente insignificante, pero que mantuve en secreto hasta que elaboré esta pieza) me ensucio en el origen cotidiano, rutinario y tabú de la violencia sexual ${ }^{47}$.

Elle instrumentalise son corps par le truchement duquel elle retrouve la notion de sacrifice liée à la passion du Christ, une figure que l'on retrouve à la fois sous des traits masculins et féminins. Dans ses créations, nourries de références religieuses, elle montre une certaine prédilection pour la mise en scène de couples, les Adam et Eve des temps modernes. Dès sa première création, elle met en exergue la souffrance des femmes dont l'origine remonte à la genèse du monde lorsque Dieu chassa des jardins d'Eden la femme qui, dès lors, endossa le rôle de la pécheresse, condamnée au châtiment éternel. Cependant, ce ne sont pas les vierges qui intéressent la dramaturge mais bien plutôt les Marie-Madeleine, les femmes mises au ban de la société. En revanche,

47. Liddell Angélica, La Desobediencia : Yo no soy bonita, In Ubu. Site de l'Université de Burgos, 2009. [En ligne]. Universidad de Burgos (ubu), 2012 [consulté le 24 octobre 2010]. Disponible sur: http://www.ubu.es/es/escena-abierta/companias-participantes/angelica-liddelldesobediencia-bonita 
elle refuse le rôle de disciple, préférant celui de rebelle : "Job se rebela contra Dios y le interroga, le pregunta ¿ por qué ?, yo me hago constantemente esa pregunta para seguir con vida, para rebelarme contra la muerte ${ }^{48} "$. Elle imagine exclusivement des personnages de antihéros, des faibles, des médiocres, comme elle-même se définit dans ¿ Cómo explicarle a un bebé la pasión anotada de Nubila Walhleim ${ }^{249}$ Dans ce texte aux allures de manifeste, elle dénigre son travail dramaturgique et ne cesse de répéter qu'il est sans intérêt, qu’il ne vaut rien : "Yo quería escribir algo bueno, algo bello ${ }^{50}$. Elle reprend des passages de ses pièces précédentes, des confessions qu'elle avait livrées au public et elle revient sur son travail dramaturgique et scénographique. Considérant son travail d'écriture comme un véritable échec, elle se lance dans l'exploration d'une nouvelle ligne de création : "Sólo me queda la oportunidad de lo confesional, lo asquerosamente confesional. [...] A partir de ahora ausencia total de personajes, lo asquerosamente confesional, solamente $^{51}$ ". Dans ce récit, Angélica Liddell déprise son travail, éclabousse son image, s'auto-détruit. Cynique, elle se lance dans de farouches critiques qui ressemblent étrangement à celles qu'elle a parfois subies : "Me siento muy porno y muy puta. Las exhibiciones del yo son odiosas ${ }^{52}$ ". En choisissant de construire ses derniers spectacles sur des épisodes de sa vie privée, elle la livre en pâture au public, s'expose jusqu'à l'indécence et déclare : "Empecé a sentir una falta absoluta de interés por el placer y un fanatismo compulsivo por el dolor ${ }^{3}$."

Enfin, même si elle se plaît à contrôler les moindres détails de ses montages, elle ne renonce jamais à l'improvisation car elle ne répète pas l'intégralité des textes. Elle les entrelace, les met en forme et en scène pour qu'ils reflètent au plus près, le cheminement de sa quête d'esthétique. Dans El matrimonio Palavrakis, Elsa clamait que toute sa vie, elle s'était évertuée à ne pas ressembler à ses parents. Depuis ses débuts, Angélica Liddell fait en sorte de ne ressembler à personne. Elle se crée une nouvelle identité, symbolisée, en premier lieu, par le patronyme qu'elle a choisi de substituer au sien. En outre, le corps devient vecteur de la pensée et il lui permet de s'exprimer, de se confronter à la société et à l'autre. Selon la créatrice, "el cuerpo es lo único que produce la verdad ${ }^{54}$ ". Ce corps, en effet, est exposé, malmené ; miroir d'aberrations il est également un révélateur de beauté et de poésie. Moyen de protestation, le corps est celui qui véhicule un discours et le point de vue de l'artiste. Les nombreuses créations,

48. Liddell Angélica, Tengo una inclinación natural a hablar de la parte podrida de las cosas. In elcultural.es. Site du journal. [En ligne]. El Cultural, 2006 [consulté le 24 octobre 2009]. Disponible sur: http://www.elcultural.es/version_papel/ESCENARIOS/26009/Angelica_ Liddel

49. Angélica Liddell, "¿ Cómo explicarle a un bebé la pasión anotada de Nubila Walhleim ?», Monólogo necesario para la extinción de Nubila Walhleim y extinción, Bilbao, Artezblai, 2009, p. 83.

50. Op. cit. (44), p. 8.

51. Op. cit. (44), p. 93.

52. Op. cit. (44), p. 63.

53. Op.cit. (44), p. 82.

54. Angélica Liddell, Perro muerto en tintorería : los fuertes, Madrid, Nórdica Libros, 2008, p. 217. 
où la provocation règne en maître, ces "actes de résistance" que revendique la dramaturge, sont les caractéristiques de son théâtre de la douleur, à la fois poétique, politique et esthétique.

\section{Bibliographie}

Artaud Antonin, Le théâtre et son double, Paris, Gallimard, 1964.

Cornago Bernal Óscar (ed.lit.), Politicas de la palabra, Madrid, Espiral/Fundamentos, 2005.

- Atra Bilis o el rito de la perversión. In Archivo Virtual artes escénicas. Site de l'Université de Castilla la Mancha. UCLM 2005 [Consulté le 24 septembre 2010]. Disponible sur http://www.artesescenicas.uclm.es/index.php?sec=texto\&id=25

Mi muerte a cambio de tu pelo. In Archivo Virtual artes escénicas. Site de l'Université de Castille la Mancha. [En ligne] UCLM, 2009 [Consulté le 24 septembre 2010]. Disponible sur : http://www.artesescenicas.uclm.es/index.php?sec=texto\&id=253

Kantor Tadeusz, Teatro de la muerte y otros ensayos 1944-1986, Barcelona, Alba Editorial, 2010.

Romera Castillo José (ed.), Análisis de espectáculos teatrales (2000-2006), Madrid, Visor Libros, 2007.

- Tendencias Escénicas al inicio del siglo XXI, Madrid, Visor Libros, 2006.

Tytell John, The Living Theatre, Arte, exilio y escándalo, Barcelona, Los Libros de la Liebre de Marzo, 1999. 